\title{
Changes in markers of fatigue following a competitive match in elite academy rugby union players
}

\author{
G Roe, ${ }^{1,2}$ MSc; K Till, ${ }^{1,2}$ PhD; J Darrall-Jones, ${ }^{1,2}$ MSc; \\ P Phibbs, ${ }^{1,2}$ MSc; J Weakley, ${ }^{1,2}$ MSc; D Read, ${ }^{1,2}$ MSc; B Jones, ${ }^{1,2} \mathrm{PhD}$ \\ ${ }^{1}$ Research Institute for Sport, Physical Activity and Leisure, Leeds Beckett \\ University, Leeds, West Yorkshire, United Kingdom \\ ${ }^{2}$ Yorkshire Carnegie Rugby Club, Headingley Carnegie Stadium, St. Michael's \\ Lane, Leeds, West Yorkshire, United Kingdom.
}

Correspondingauthor: GRoe (g.roe@leedsbeckett.ac.uk)

Background: Post-match fatigue has yet to be investigated in academy rugby union players.

Objectives: To determine the magnitude of change in upper(plyometric push-up (PP) flight-time) and lower-body (countermovement jump (CMJ) mean power) neuromuscular function (NMF), whole blood creatine kinase (CK) and perception of well-being following a competitive match in academy rugby union players.

Methods: Fourteen academy rugby union players participated in the study. Measures were taken $2 \mathrm{~h}$ pre-match (baseline) and immediately post-match. Further testing was also undertaken at 24-, 48- and $72 \mathrm{~h}$ respectively post-match. Changes in measures from baseline were determined using magnitude-based inferences. Results: Decreases in CMJ mean power were likely substantial immediately $(-5.5 \pm 3.3 \%)$ post-match, very likely at $24 \mathrm{~h}(-7 \pm 3.9)$, likely at $48 \mathrm{~h}(-5.8 \pm 5.4)$, while likely trivial at $72 \mathrm{~h}(-0.8 \pm 3.8)$ post-match. PP flight-time was very likely reduced immediately $(-15.3 \pm 7.3 \%)$ and $24 \mathrm{~h}(-11.5 \pm 5.7 \%)$ post-match, while possibly increased at $48 \mathrm{~h}(3.5 \pm 6.0 \%)$ and likely trivial at $72 \mathrm{~h}(-0.9 \pm 5.4 \%)$ post-match. Decreases in perception of well-being were almost certainly substantial at $24 \mathrm{~h}(-24.0 \pm 4.3 \%)$, very likely at $48 \mathrm{~h}$ $(-8.3 \pm 5.9 \%)$, and likely substantial at $72 \mathrm{~h}(-3.6 \pm 3.7 \%)$ post-match. Increases in CK were almost certainly substantial immediately (138.5 $\pm 33 \%), 24 \mathrm{~h}(326 \pm 78 \%)$ and $48 \mathrm{~h}(176 \pm 62 \%)$ post-match, while very likely substantial at $72 \mathrm{~h}(57 \pm 35 \%)$ post-match.

Conclusion: These findings demonstrate the transient and multidimensional nature of post-match fatigue in academy rugby union players. Furthermore, the results demonstrate the individual nature of recovery, with many players demonstrating different recovery profiles from the group average. Keywords: collision sport, monitoring, sports injuries

S Afr J Sports Med 2016;28(1):2-5. DOI:10.17159/2078-516X/2016/v2811a418

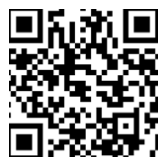

Rugby union is a collision sport that involves intermittent high-intensity activities, including sprinting, rucking, mauling, scrummaging and tackling, that are interspersed with periods of jogging, walking and standing. ${ }^{[1]}$ Observations following one, ${ }^{[2,3]}$ two $^{[4]}$ and four ${ }^{[5]}$ competitive matches suggest that the highintensity activities and impacts sustained during rugby union match play result in acute post-match fatigue that may last for several days following competition. Fatigue may manifest as alterations in mood, ${ }^{[3]}$ immune function ${ }^{[2]}$ and hormone levels, ${ }^{[3]}$ reductions in neuromuscular function $(\mathrm{NMF})^{[3]}$ and elevations in markers of muscle damage (e.g. an increase in creatine kinase concentration $\mathrm{CK}$ ). ${ }^{[2,5]}$
The authors' understanding of post-match fatigue in rugby union players has been derived primarily from studies involving senior athletes. To date, no study has investigated post-match fatigue in academy rugby union players. The literature examining postmatch fatigue in other junior collision sport athletes is also far less voluminous than in senior players. A likely substantial reduction in lower-body NMF, as measured by countermovement jump (CMJ) peak power, has been observed in elite under-18 Australian rules football players for up to $24 \mathrm{~h}$ following an intra-club preseason match $^{[6]}$ while in sub-elite youth rugby league players, reductions were reported as likely substantial immediately, and possibly substantial at $24 \mathrm{~h}$ and $48 \mathrm{~h}$ following two competitive matches. ${ }^{[7]}$ Upper-body NMF, as measured by plyometric push-up peak power, has also shown very likely and likely substantial decreases at $24 \mathrm{~h}$ and $48 \mathrm{~h}$ respectively following two competitive sub-elite youth rugby league matches. ${ }^{[7]}$ Furthermore, CK has been observed to peak at $24 \mathrm{~h}$ post-match and remain elevated at $48 \mathrm{~h}$ in sub-elite junior rugby league players. ${ }^{[7]}$ However, no study has examined CK responses to match-play in this population beyond $48 \mathrm{~h}$ post-match.

Understanding the time-course of recovery following a competitive match is extremely important. Such knowledge can be applied to ensure that players have adequately recovered prior to undertaking subsequent training or competing in a later game. Failure to recover may lead to fatigue accumulation and result in injury, illness and poor performance. ${ }^{[2,8]}$ Research in academy rugby union players provides scientific evidence with which to inform recovery and training practices in the post-match microcycle in this group of athletes. Therefore the purpose of the current study was to investigate the magnitude of change, and the time-course of recovery in markers of NMF, muscle damage and perception of well-being following a competitive match in academy rugby union players.

\section{Methods}

\section{Subjects}

Fourteen players (age $17.4 \pm 0.8$ years; height $182.7 \pm 7.6 \mathrm{~cm}$; body mass $86.2 \pm 11.6 \mathrm{~kg}$ ) were recruited from a professional rugby union academy. Players were excluded if they had an injury that prevented them from participating in the testing or were involved in less than $75 \%$ of the total game time. The University's Ethics Board granted approval and written informed consent was acquired from all subjects along with parental consent.

\section{Procedures and design}

A within-group repeated measures design was used to examine the magnitude of change in markers of NMF, muscle damage (whole blood CK) and perception of well-being following a competitive match between academy rugby union players. Lower-body NMF was measured using a countermovement jump (CMJ), while upperbody NMF was measured using a plyometric push-up. The extent of muscle damage was examined by measuring changes in plasma $\mathrm{CK}$ and perception of well-being was quantified by means of a questionnaire. Measures of CMJ, plyometric push-up, plasma CK and perception of well-being were taken two hours pre-match (baseline) 
and immediately post-match. Further testing was also undertaken at $24 \mathrm{~h}, 48 \mathrm{~h}$ and $72 \mathrm{~h}$ post-match at the same time of day as baseline measures to avoid diurnal effects on performance. During the testing period, players did not engage in any training or strenuous activity in the days following the match. Players were advised on nutritional intake but no recovery protocol was undertaken.

\section{Neuromuscular function}

Lower-body NMF was measured using mean power calculated from a CMJ, while upper-body NMF was measured using flight-time calculated from a plyometric push-up. Both of these measures have previously been proven reliable in this population (typical error $=$ $3.1 \%$ and $4.2 \%$ respectively). ${ }^{[9]}$ The CMJ and plyometric push-up were performed on a portable force plate (400 Series Performance Plate, Fitness Technology, Adelaide, Australia) that was attached to a laptop with software (Ballistic Measurement System, Fitness Technology, Adelaide, Australia) that measured ground reaction forces at $600 \mathrm{~Hz}$. A standardised two-minute warm-up consisting of dynamic stretching was performed prior to the performance tests (walking lunges, squats, heel flicks, high knees, skipping, leg swings and three practice submaximal CMJ and plyometric pushups). Following the warm-up, players performed two maximal CMJ followed by two maximal plyometric push-ups with a one-minute rest between each effort. ${ }^{[9]}$

For the CMJ, players began standing on the force platform with knees extended and feet in a position of their choice. Players were instructed to keep their hands on their hips and jump as a high as possible. The depth of the countermovement was at the discretion of the subject. ${ }^{[9]}$ For the plyometric push-up, Players began with their elbows extended and hands on the force platform in a position of their choice. Players were instructed to perform a push-up as quickly as possible ensuring that their hands left the platform. ${ }^{[7]}$

\section{Perception of well-being}

A six-item questionnaire was adapted from McLean et al. ${ }^{[10]}$ to rate the following: sleep, fatigue, muscle soreness (upper- and lowerbody), stress and mood on a five-point Likert scale. Each item was rated from one to five in one score increments and overall well-being was assessed by adding up all six scores. Reliability of this method has previously been reported $(\mathrm{CV}=7.1 \%) .{ }^{[9]}$ The questionnaire was administered prior to any other testing being undertaken. ${ }^{[10]}$ Subjects completed the questionnaire on their own in order to prevent any influence from other players. ${ }^{[1]}$

\section{Creatine kinase}

Whole blood samples were collected from the non-dominant hand, middle fingertip of each subject. Approximately $30 \mu$ lof whole capillary blood was collected using a plastic capillary tube (MICROSAFE ${ }^{\bullet}$, Safe-tec, Numbrecht, Ivyland, USA) and immediately analysed using reflectance photometry (Refletron ${ }^{\star}$ Plus, Boehringer Manheim, Germany). Prior to each session, the machine was calibrated using a standardised CK strip to ensure that the machine was analysing correctly. The reliability of this method has previously been reported $(\mathrm{CV}=26.1 \%) .{ }^{[9]}$

\section{Match demands}

External match loads were assessed using GPS and accelerometer technology (Optimeye S5, Catapult Innovations, Melbourne, Australia). Based on individual maximum velocities established three weeks prior to the match, locomotive demands were classified for each player as: walking and standing $(<20 \%$ Vmax $)$, jogging (20-50\% Vmax), striding (51-80\% Vmax), sprinting (81-95\% Vmax) and maximum sprinting $(96-100 \% \operatorname{Vmax})^{[1]}$. However, as little distance was covered at maximum sprinting speed $(1.43 \pm 4.01 \mathrm{~m})$, the sprinting and maximum sprinting categories were aggregated to form one sprinting category (81-100\% Vmax). Internal load was established using the session rating of perceived exertion method $(\mathrm{sRPE})^{[12]}$ within 15-30 minutes of the match on a modified Borg scale.

\section{Statistical analysis}

Pre- and post-match NMF and CK were log transformed to reduce bias as a result of non-uniformity error. Perceptual data was analysed in its raw form. Data were all analysed for practical significance using magnitude-based inferences. ${ }^{[13]}$ The threshold for a change to be considered practically important (the smallest worthwhile change; SWC) was set at $0.2 \mathrm{x}$ between subject standard deviation (SD), based on Cohen's d effect size (ES) principle. The probability that the magnitude of change was greater than the SWC was rated as $<0.5 \%$, almost certainly not; $0.5-5 \%$, very unlikely; $5-25 \%$, unlikely; 25-75\%, possibly; 75-95\%, likely; 95-99.5\%, very likely; >99.5\%, almost certainly. ${ }^{[13]}$ Where the $90 \%$ confidence interval (CI) crossed both the upper and lower boundaries of the SWC $(\mathrm{ES} \pm 0.2)$, the magnitude of change was described as unclear. ${ }^{[13]}$

\section{Results}

The duration of the match was 73:37 minutes. The first and second halves lasted 36:30 minutes and 37:07 minutes, respectively. The average match load (Rate of Perceived Exertion (RPE) x time)) was $334 \pm 121$ arbitrary units (AU). Players covered $4691 \pm 878 \mathrm{~m}$ during the match with an average of $74 \pm 6 \mathrm{~m} \cdot \mathrm{min}^{-1}$. Of the total distance, $1771 \pm 436 \mathrm{~m}$ was covered walking / standing, $2215 \pm 461 \mathrm{~m}$ jogging, $663 \pm 238 \mathrm{~m}$ striding and $41 \pm 40 \mathrm{~m}$ sprinting. Average Player Load ${ }^{\mathrm{TM}}$ was $451 \pm 102$ and Player Load ${ }^{\mathrm{TM}}$ slow was $187 \pm 47$.

\section{Lower-body neuromuscular function}

Decreases in CMJ mean power were likely substantial immediately $(-5.5 \pm 3.3 \%)$ post-match, very likely at $24 \mathrm{~h}(-7.0 \pm 3.9)$, likely at $48 \mathrm{~h}(-5.8 \pm 5.4)$, while likely trivial at $72 \mathrm{~h}(-0.8 \pm 3.8)$ post-match (Figure 1A).

\section{Upper-body neuromuscular function}

Reductions in plyometric push-up flight-time were very likely substantial immediately $(-15.3 \pm 7.3 \%)$ and $24 \mathrm{~h}(-11.5 \pm 5.7 \%)$ postmatch, while there was a possible increase at $48 \mathrm{~h}(3.5 \pm 6 \%)$ post-match and trivial changes at $72 \mathrm{~h}(-0.9 \pm 5.4 \%)$ post-match (Figure $1 \mathrm{~B})$.

\section{Perception of well-being}

Decreases in perception of well-being were almost certainly substantial at $24 \mathrm{~h}(-24 \pm 4.3 \%)$, very likely at $48 \mathrm{~h}(-8.3 \pm 5.9 \%)$, and likely at $72 \mathrm{~h}(-3.6 \pm 3.7 \%)$ post-match (Figure $1 \mathrm{C})$. 


\section{Creatine kinase}

Increases in CK were almost certainly substantial immediately $(138.5 \pm 33.1 \%), 24 \mathrm{~h}(326 \pm 77.6 \%)$ and $48 \mathrm{~h}(176.4 \pm 62.4 \%)$ postmatch, while very likely substantial at $72 \mathrm{~h}(56.7 \pm 34.5 \%)$ post-match (Figure 1D).

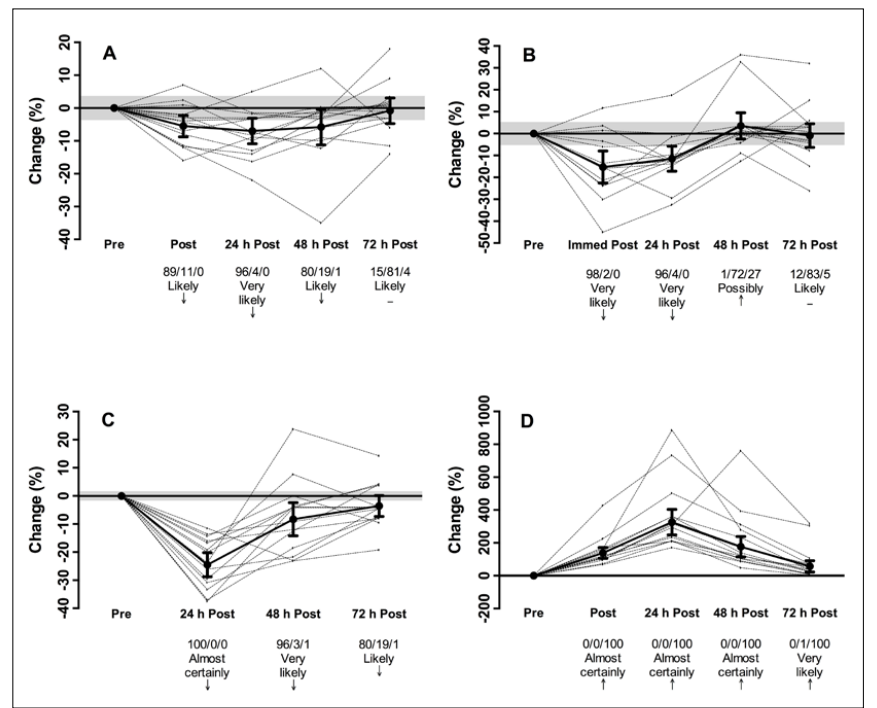

Fig. 1. Percentage changes in CMJ mean power (A), plyometric push-up flight-time (B), well-being $(C)$ and whole blood creatine kinase concentration $(D)$.

Data are percentage change with dotted and continuous lines representing individual changes and group mean changes with $90 \%$ confidence interval bars respectively, and the shaded area representing the smallest worthwhile change as a percentage. Probabilities of a decrease / trivial / increase values; increase $\uparrow$ decrease $\downarrow$ trivial -.

\section{Discussion}

The present study demonstrates the time-course of recovery in markers of fatigue following a competitive match in academy rugby union players. Reductions in lower-body neuromuscular function were likely to very likely substantial for up to $48 \mathrm{~h}$ post-match, but likely trivial by $72 \mathrm{~h}$ post-match. Similar changes have been observed in CMJ peak power in senior players for up to $36 \mathrm{~h}$ following a competitive match. ${ }^{[3]}$ Comparable findings have also been reported in other junior collision-sport athletes. For example, Wehbe et al. ${ }^{[6]}$ noted very likely substantial reductions in CMJ mean power for up to $24 \mathrm{~h}$ in under-18 Australian rules football players following an intra-club preseason match, although changes were unclear beyond this point. Johnston and colleagues ${ }^{[7]}$ also observed likely substantial reductions in peak power immediately post-match and possible reductions at 24 - and $48 \mathrm{~h}$ following two competitive matches in subelite youth rugby league players.

The post-match transient reductions in NMF may be the result of central fatigue, resulting in a reduction in voluntary muscle activation. ${ }^{[14]}$ Furthermore, muscle damage from repeated stretchshortening cycle actions that occur during high-intensity running, ${ }^{[5]}$ and also from the blunt trauma to the lower-limb musculature that occurs during collisions, ${ }^{[11]}$ may have further contributed to the reduction in NMF.
In contrast, upper-body NMF in the present study was very likely reduced for $24 \mathrm{~h}$ post-match, with substantial reductions being unlikely beyond this point. This reduction may be attributed to the blunt trauma sustained during physical contact, which has a substantial effect on upper-body NMF. ${ }^{[7]}$ Changes in upper-body NMF have not currently been investigated in senior rugby union players. However, in the previously mentioned study involving subelite rugby league players by Johnston and colleagues ${ }^{[7]}$ almost certain substantial reductions in PP peak power were observed immediately post-match, while likely substantial reductions were reported 24 and $48 \mathrm{~h}$ post-match. Unfortunately a direct comparison with the present study cannot be made, as this metric was not included in the analysis due to unacceptable reliability in this population. ${ }^{[9]}$

Perception of well-being demonstrated almost certain substantial reductions at $24 \mathrm{~h}$ post-game and remained very likely and likely substantially reduced at $48 \mathrm{~h}$ and $72 \mathrm{~h}$ post-match. In senior collisionsport athletes, reductions in perception of well-being have been reported to peak at $24 \mathrm{~h}$ post-match and gradually return to baseline thereafter ${ }^{[11]} \mathrm{A}$ similar trend occurred in the present study with only small $(-3.6 \pm 3.7 \%)$ decreases being observed by $72 \mathrm{~h}$ post-match.

In the present study, CK peaked in the first $24 \mathrm{~h}$ post-match, which is in accordance with findings from other studies in both junior ${ }^{[7]}$ and senior ${ }^{[5,11]}$ collision sport athletes. Elevations in CK have been associated with damage to skeletal muscle tissue, either from direct trauma during collisions ${ }^{[4]}$ or repetitive eccentric damage during high-speed running ${ }^{[5,11]}$ throughout match-play. The resulting disruption of skeletal muscle integrity leads to leakage of CK into the bloodstream. ${ }^{[15]}$ The results demonstrate that biochemical homeostasis had not been restored by $72 \mathrm{~h}$, with CK levels still very likely substantially elevated $(56.7 \pm 34.5 \%)$ at this time. However, the consequence of this is unclear. Given that lower-body neuromuscular recovery was evident by $72 \mathrm{~h}$, the large elevation in CK at this time may reflect the rate of CK clearance from the blood, ${ }^{[15]}$ and not the extent of muscle damage.

The results of the present study also demonstrate the individual nature of recovery following match-play in academy rugby union players. Figure 1 shows that even when the group mean demonstrated near full recovery, certain individuals still exhibited negative changes that were greater than the SWC. These findings emphasise that although understanding a group response provides valuable information on the recovery in the days post-match, it is important for practitioners to monitor the recovery of each individual player following competition.

A limitation of the present study is the analysis of only one competitive match. However, the total distance covered in the current study $(4691 \pm 878 \mathrm{~m})$ was similar to the average distances reported from five games $(4470 \pm 292 \mathrm{~m})$ by Venter et al. ${ }^{[16]}$ in under-19 provincial players, suggesting this may represent academy rugby match demands. However, future research involving a greater number of observations is needed to further investigate the specific demands of match-play that result in post-match fatigue in academy rugby union players. Furthermore, such research may also provide insight into positional differences, both in terms of the specific match demands that cause fatigue, and the magnitude of fatigue following match play. 


\section{Conclusion}

The findings of the present study demonstrate the transient and multidimensional nature of post-match fatigue in academy rugby union players. A decrease in upper-body NMF was very likely for up to $24 \mathrm{~h}$ but was unlikely beyond this point, while lower-body NMF was still likely decreased at $48 \mathrm{~h}$ before returning to baseline. In contrast, perception of well-being and CK were negatively altered from immediately to $72 \mathrm{~h}$ post-match, although returning towards baseline at this time. Furthermore, the results demonstrate the individual nature of recovery, with many players demonstrating different recovery profiles from the group average.

\section{References}

1. Cahill N, Lamb K, Worsfold P, et al. The movement characteristics of English Premiership rugby union players. J Sports Sci 2013;31(3):229-237. [http://dx.doi.org /10.1080/02640414.2012.727456]

2. Cunniffe B, Hore AJ, Whitcombe DM, et al. Time course of changes in immuneoendocrine markers following an international rugby game. Eur J Appl Physiol 2010;108(1):113-122. [http://dx.doi.org/10.1007/s00421-009-1200-9]

3. West DJ, Finn CV, Cunningham DJ, et al. Neuromuscular function, hormonal, and mood responses to a professional rugby union match. J Strength Cond Res 2014;28(1):194-200. [http://dx.doi.org/10.1519/JSC.0b013e318291b726]

4. Takarada Y. Evaluation of muscle damage after a rugby match with special reference to tackle plays. Br J Sports Med 2003;37(5):416-419. [http://dx.doi.org/10.1136/ bjsm.37.5.416]

5. Jones $\mathrm{M}$, West $\mathrm{D}$, Harrington $\mathrm{B}$, et al. Match play performance characteristics that predict post-match creatinekinase responses in professional rugby union players. BMC Sports Sci Med Rehabil 2014;6(1):38. [http://dx.doi.org/10.1186/2052-1847-6-38]

6. Wehbe G, Gabett TJ, Dwyer D, et al. Monitoring neuromuscular fatigue in team-sport athletes using a cycle-ergometer test. Int J Sports Physiol Perform 2015;10(3):292-297. [http://dx.doi.org/10.1123/ijspp.2014-0217]
7. Johnston RD, Gabbett TJ, Jenkins DG, et al. Influence of physical qualities on postmatch fatigue in rugby league players. J Sci Med Sport 2015;18(2):209-213. [http:// dx.doi.org/10.1016/j.jsams.2014.01.009]

8. Johnston RD, Gabbett TJ, Jenkins DG. Influence of an intensified competition on fatigue and match performance in junior rugby league players. J Sci Med Sport 2013;16(5):460-465. [http://dx.doi.org/10.1016/j.jsams.2012.10.009]

9. Roe G, Darrall-Jones J, Till K, et al. Between-day reliability and sensitivity of common fatigue measures in rugby players. Int J Sports Physiol Perform 2015, Sep 21. [Epub ahead of print] [http://dx.doi.org/10.1123/ijspp.2015-0413]

10. McLean BD, Coutts AJ, Kelly V, et al. Neuromuscular, endocrine, and perceptual fatigue responses during different length between-match microcycles in professional rugby league players. Int J Sports Physiol Perform 2010;5(3):367-383. [http://dx.doi. org/10.1123/ijspp.5.3.367]

11. Twist C, Waldron M, Highton J, et al. Neuromuscular, biochemical and perceptual post-match fatigue in professional rugby league forwards and backs. J Sports Sci 2012;30(4):359-367. [http://dx.doi.org/10.1080/02640414.2011.640707]

12. Foster C, Florhaug JA, Franklin J, et al. A new approach to monitoring exercise training. J Strength Cond Res. 2001;15(1):109-115. [http://dx.doi.org/ 10.1519/00124278-200102000-00019]

13. Hopkins WG, Marshall SW, Batterham AM, et al. Progressive statistics for studies in sports medicine and exercise science. Med Sci Sports Exerc. 2009;41(1):3-13 [http:// dx.doi.org/10.1249/MSS.0b013e31818cb278]

14. Boyas S, Guével A. Neuromuscular fatigue in healthy muscle: Underlying factors and adaptation mechanisms. Annals Phys Rehabil Med 2011;54(2):88-108. [http:// dx.doi.org/10.1016/j.rehab.2011.01.001]

15. Baird MF, Graham SM, Baker JS, et al. Creatine-kinase- and exercise-related muscle damage implications for muscle performance and recovery. J Nutr Metabolism 2012; 2012:960363. [http://dx.doi.org/10.1155/2012/960363]

16. Venter RE, Opperman E, Opperman S. The use of global positioning system (GPS) tracking devices to assess movement demands and impacts in under-19 rugby union match play. Afr J Phys Health Ed Rec Dance 2011;17(1):1-8. [http://dx.doi. org/10.4314/ajpherd.v17il.65242] 